\title{
Comprehensive analysis and establishment of a prediction model of alternative splicing events reveal the prognostic predictor and immune microenvironment signatures in triple negative breast cancer
}

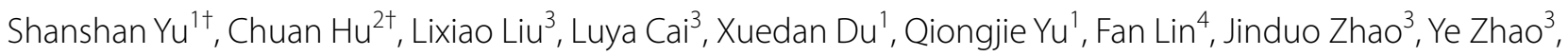
Cheng Zhang ${ }^{4}$, Xuan Liü and Wenfeng $\mathrm{Li}^{3}{ }^{*}$ (D)

\begin{abstract}
Background: Triple-negative breast cancer (TNBC) is widely concerning because of high malignancy and poor prognosis. There is increasing evidence that alternative splicing (AS) plays an important role in the development of cancer and the formation of the tumour microenvironment. However, comprehensive analysis of AS signalling in TNBC is still lacking and urgently needed.

Methods: Transcriptome and clinical data of 169 TNBC tissues and 15 normal tissues were obtained and integrated from the cancer genome atlas (TCGA), and an overview of AS events was downloaded from the SpliceSeq database. Then, differential comparative analysis was performed to obtain cancer-associated AS events (CAAS). Metascape was used to perform parent gene enrichment analysis based on CAAS. Unsupervised cluster analysis was performed to analyse the characteristics of immune infiltration in the microenvironment. A splicing network was established based on the correlation between CAAS events and splicing factors (SFs). We then constructed prediction models and assessed the accuracy of these models by receiver operating characteristic (ROC) curve and Kaplan-Meier survival analyses. Furthermore, a nomogram was adopted to predict the individualized survival rate of TNBC patients.

Results: We identified 1194 cancer-associated AS events (CAAS) and evaluated the enrichment of 981 parent genes. The top 20 parent genes with significant differences were mostly related to cell adhesion, cell component connection and other pathways. Furthermore, immune-related pathways were also enriched. Unsupervised clustering analysis revealed the heterogeneity of the immune microenvironment in TNBC. The splicing network also suggested an obvious correlation between SFs expression and CAAS events in TNBC patients. Univariate and multivariate Cox regression analyses showed that the survival-related AS events were detected, including some significant participants in the carcinogenic process. A nomogram incorporating risk, AJCC and radiotherapy showed good calibration and moderate discrimination.
\end{abstract}

\footnotetext{
*Correspondence: Iwf720325@126.com

†Shanshan Yu and Chuan Hu are contributed equally to this work

${ }^{1}$ Department of Chemoradiation Oncology, The First Affiliated Hospital

of Wenzhou Medical University, 2 Fuxue Road, Wenzhou, Zhejiang

325000, People's Republic of China

Full list of author information is available at the end of the article
}

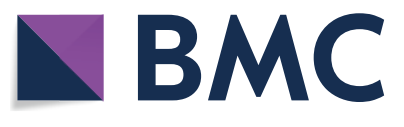

(c) The Author(s) 2020. This article is licensed under a Creative Commons Attribution 4.0 International License, which permits use, sharing, adaptation, distribution and reproduction in any medium or format, as long as you give appropriate credit to the original author(s) and the source, provide a link to the Creative Commons licence, and indicate if changes were made. The images or other third party material in this article are included in the article's Creative Commons licence, unless indicated otherwise in a credit line to the material. If material is not included in the article's Creative Commons licence and your intended use is not permitted by statutory regulation or exceeds the permitted use, you will need to obtain permission directly from the copyright holder. To view a copy of this licence, visit http://creativeco mmons.org/licenses/by/4.0/. The Creative Commons Public Domain Dedication waiver (http://creativecommons.org/publicdomain/ zero/1.0/) applies to the data made available in this article, unless otherwise stated in a credit line to the data. 
Conclusion: Our study revealed AS events related to tumorigenesis and the immune microenvironment, elaborated the potential correlation between SFs and CAAS, established a prognostic model based on survival-related AS events, and created a nomogram to better predict the individual survival rate of TNBC patients, which improved our understanding of the relationship between AS events and TNBC.

Keywords: Alternative splicing, TNBC, Nomogram, Prognosis, Immune signatures

\section{Introduction}

Breast cancer is the most common cancer among women in the world, with the highest incidence, and is the second leading cause of cancer-related death, which has strong impacts on national economic and social development [1]. There are many kinds of breast cancers, among which triple negative breast cancer (TNBC) is a wellknown subtype with high invasion, a low survival rate and a lack of effective treatment, accounting for 15-20\% of all breast cancers and becoming an intractable problem in the breast cancer field [2]. The clinical manifestation of TNBC is an aggressive course, which is prone to local recurrence and distant metastasis. Once there is recurrence or metastasis, the median survival time is less than 1 year $[3,4]$. The anti-programmed cell death (PD)-1 immunotherapy has taken on a favourable effect in TNBC according to the results of recent research, but the efficacy rate of these drugs is still low [5-7]. The poor prognosis of TNBC is closely related to its early high metastasis rate and recurrence rate [8-10], which suggests the urgent need to develop new biomarkers with high accuracy to predict the prognosis of TNBC patients.

In recent years, research on cancer genomics has gradually opened a new chapter benefiting from the tremendous development of bioinformatics and highthroughput technologies. A growing body of evidence shows that alternative splicing (AS) events play a pivotal role in the development of cancer and the formation of the tumour microenvironment $[11,12]$. AS events have great influence in the process of mRNA precursor maturation, bringing about the splicing of different mRNA isomers and the translation of protein variants, which is one of the key regulatory mechanisms for the diversity of the transcriptome and proteome [13, 14]. In normal physiological processes, more than $95 \%$ of human genes undergo AS and encode splicing mutations [15]. AS not only exerts an important influence in normal physiological processes such as haematopoiesis and muscle [16, 17] but also plays a crucial role in carcinogenic pathological processes such as tumour proliferation, apoptosis, hypoxia, angiogenesis, immune escape and metastasis $[18,19]$. Moreover, protein diversity leads to functional diversity, and quantitative change causes qualitative change. The production and accumulation of irregular AS events change the expression of some key splicing factors and promote the changes in the targeted AS parent genes, which together provide an advantage for the growth or survival of cancer cells [20]. Therefore, increasing amounts of attention have been paid to research on the effect of AS events on the cancer prognosis. The comprehensive analysis of AS events is expected to provide new potential biomarkers for the diagnosis and prognosis of cancer.

The prognostic value derived from AS events has been confirmed in a variety of cancer types, including liver cancer [21], head and neck tumours [22], kidney carcinoma [23], and pancreatic cancer [24]. However, to our knowledge, comprehensive analysis of AS signals in TNBC is lacking. Because of the severe prognosis of TNBC, it is urgent and necessary to carry out relevant research. In our study, we obtained and integrated transcriptome and clinical data of 169 TNBC tissues and 15 adjacent normal tissues from the cancer genome atlas (TCGA) and downloaded the data of AS events from the SpliceSeq database. Then, several bioinformatic and statistical methods were performed to analyse the function and prognostic value of AS events in TNBC patients, which filled in the blanks of TNBC in the aspect of AS events and laid a theoretical foundation for guiding clinical work and evaluating patient prognosis.

\section{Methods}

\section{Data collection based on TCGA}

We collected the transcriptome data and the clinical information of TNBC tissues and normal breast tissues from the TCGA data portal. We also downloaded data for AS events from the TCGA SpliceSeq database. There was a broad consensus that the goal of using Percent Spliced In (PSI) [25] ranging from 0 to 1 is to quantify events. We then set a strict set of screening conditions (sample percentage with a PSI value of 75 and an average PSI value of 0.05 ) to ensure the reliability of AS events included in subsequent analysis. Ultimately, $169 \mathrm{TNBC}$ patients were included in our research.

\section{Screen for cancer-associated AS (CAAS) events between TNBC and normal tissues}

To analyse the significantly differentially expressed AS events between tumour and normal samples, 169 TNBC and 15 normal tissues were used to perform differential 
analysis using the "limma" package. We took log FC (log twofold change) and false discovery rate (FDR) as the indexes to observe the expression differences, that is, associated adj.p values. We set the condition of $\mid \log 2 \mathrm{fc}$ $\mid \geq 1$ and FDR/adjusted $P<0.05$ to represent the upregulation and downregulation of relevant events, respectively.

\section{Functional enrichment analysis and exploration of the immune microenvironment}

Based on the CAAS events, we further performed enrichment analysis of the corresponding parent genes. Gene Ontology (GO) and Kyoto Encyclopedia of Genes and Genomes (KEGG) pathway analyses were performed in metascape (www.metascape.org). The first 20 important pathways, if possible, were shown in the KEGG and GO analyses. Additionally, the TCGA TNBC cohort was classified by hierarchical consensus clustering. We employed the ConsensusClusterPlus package for the sake of clustering in an unbiased and unsupervised manner, and patients were classified into several clusters [26]. To obtain a robust classification, the optimal numbers of clusters were further validated according to the Elbow method and the Gap statistic. The differences in immune cells and the tumour immune microenvironment between the three clusters were compared by K-W test or the Wilcoxon rank-sum test.

\section{Building of potential SF-AS regulatory network}

Studies have shown that splicing factor (SF) plays an indispensable role in regulating the development and progress of malignant tumors [27, 28].We downloaded SF data from the SpliceAid2 database (https://www.intro ni.it/splicing.html).Pearson correlation analysis was used to analyze the relationship between SFs expression and PSI values of CAAS events. Absolute value of correlation coefficient $>0.5, P<0.001$ is considered to be correlated.The correlation plot was generated by Cytoscape (version3.7.2).

\section{Construction of prognostic models and survival analysis}

To further understand the prognostic value of AS events in TNBC patients, univariate Cox regression analysis was performed to determine survival-related DEAS events, including OS (overall survival)- and PFS (progressionfree survival)-related DEAS events. Meanwhile, the UpSetR package in $\mathrm{R}$ was performed to draw two UpSet diagrams to show the interactions between seven types of survival-related CAAS events [29, 30]. Next, the least absolute shrinkage and selection operator (LASSO) regression was applied to identify the final elimination of potential predictors with non-zero coefficients [31], which can avoid model overfitting to obtain a betterfitting model. Furthermore, predictive models according to the results of LASSO Cox regression were constructed using multivariate Cox regression analysis. Based on PSI values and multivariate Cox analysis, we calculated the risk scores of each patient and obtained the corresponding coefficients, respectively. Risk score can be obtained by the following formula: score $=\sum_{i=0}^{n} P S I \times \beta_{i}$, where $\beta$ is the regression coefficient.

A total of 169 TNBC patients were divided into highand low-risk groups bounded by the median of risk score, and Kaplan-Meier survival analysis was performed to determine whether they had completely different prognoses. Furthermore, receiver operating characteristic (ROC) curves of 2, 3, and 4 years were generated to show the discrimination of predictive signatures.

\section{Development of an AS-clinic nomogram}

A nomogram is an easy to use tool for clinical practice, especially in clinical oncology. Therefore, we utilized ASbased risk scores and clinical variables to develop 2 nomograms. First, we used univariate Cox analysis to screen corresponding variables related to survival, including OS and PFS. We utilized a forward stepwise variable selection with the Akaike information criterion (AIC) to filter the variables included in the final model nomogram. Finally, we created calibration curves to assess the predictive accuracy of the final nomogram and adopted the concordance index (C-index) as an index to quantify its discrimination capacity with the help of Hmisc package (version 4.1.1).

\section{Statistical analysis}

All statistical analyses were performed in R software, version 3.6.1. All statistical tests with $p<0.05$ (two-sided) were statistically significant.

\section{Results}

\section{Identification of CAAS events}

A flow chart summarizing the present work is shown in Fig. 1. We integrated the AS profiling of 169 TNBC tissues and 15 normal tissues to determine whether there were significant differences in AS events between tumour tissues and corresponding paracancerous normal tissues. Finally, we screened and identified 1194 differentially expressed CAAS events using the conditions of $\mid \log 2 \mathrm{fc}$ $\mid \geq 1$ and FDR/adjusted $P<0.05$. Meanwhile, a heat map (Fig. 2a) and a volcano plot (Fig. 2b) were generated to visualize CAAS events.

Subsequently, enrichment analysis was performed, and the results are shown in Fig. 3. The top 20 results of enrichment pathways disclosed by biological processes in GO analysis included "adherens junction", "cell cortex", and "cell adhesion molecule binding", among which the molecular functions of the cellular 

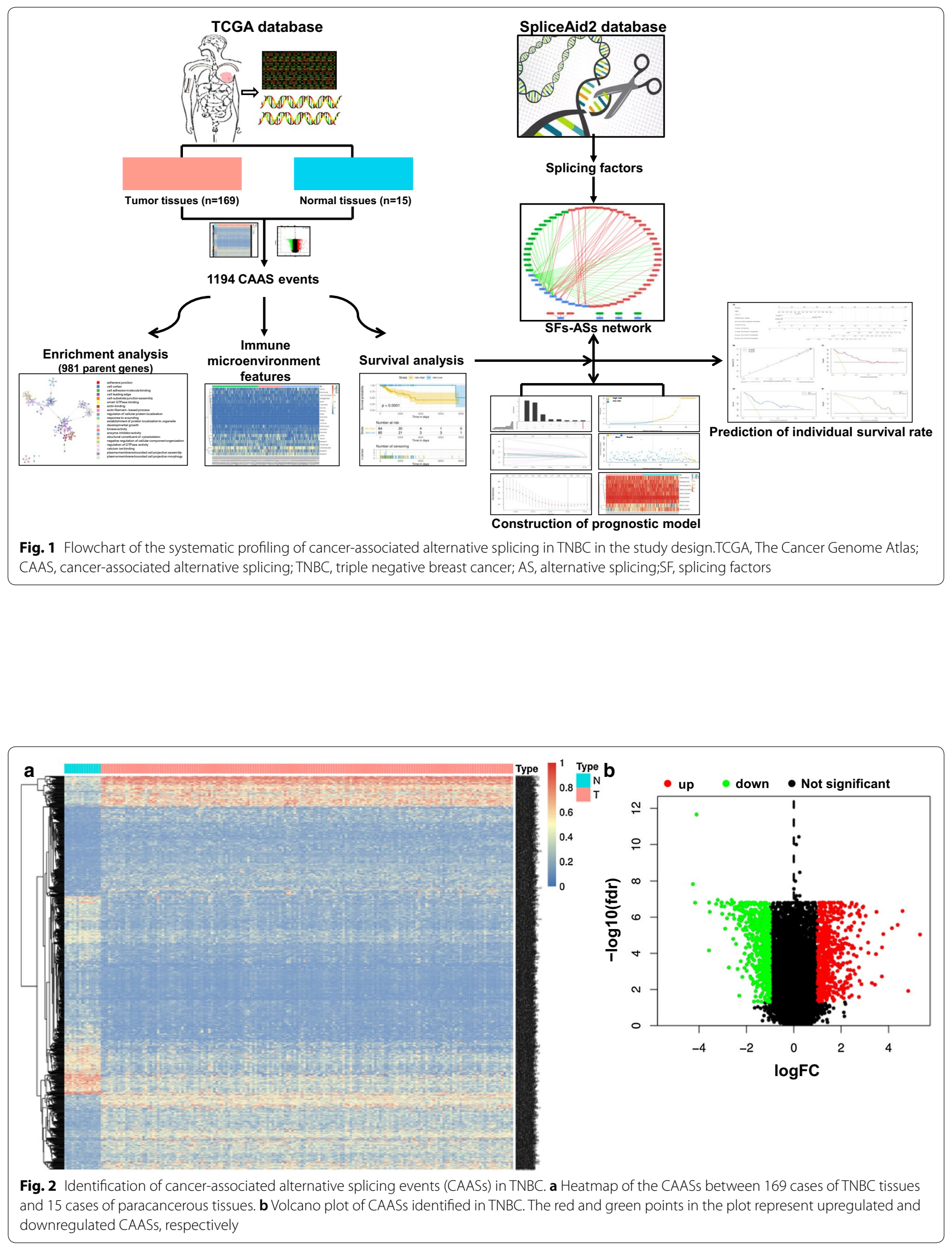


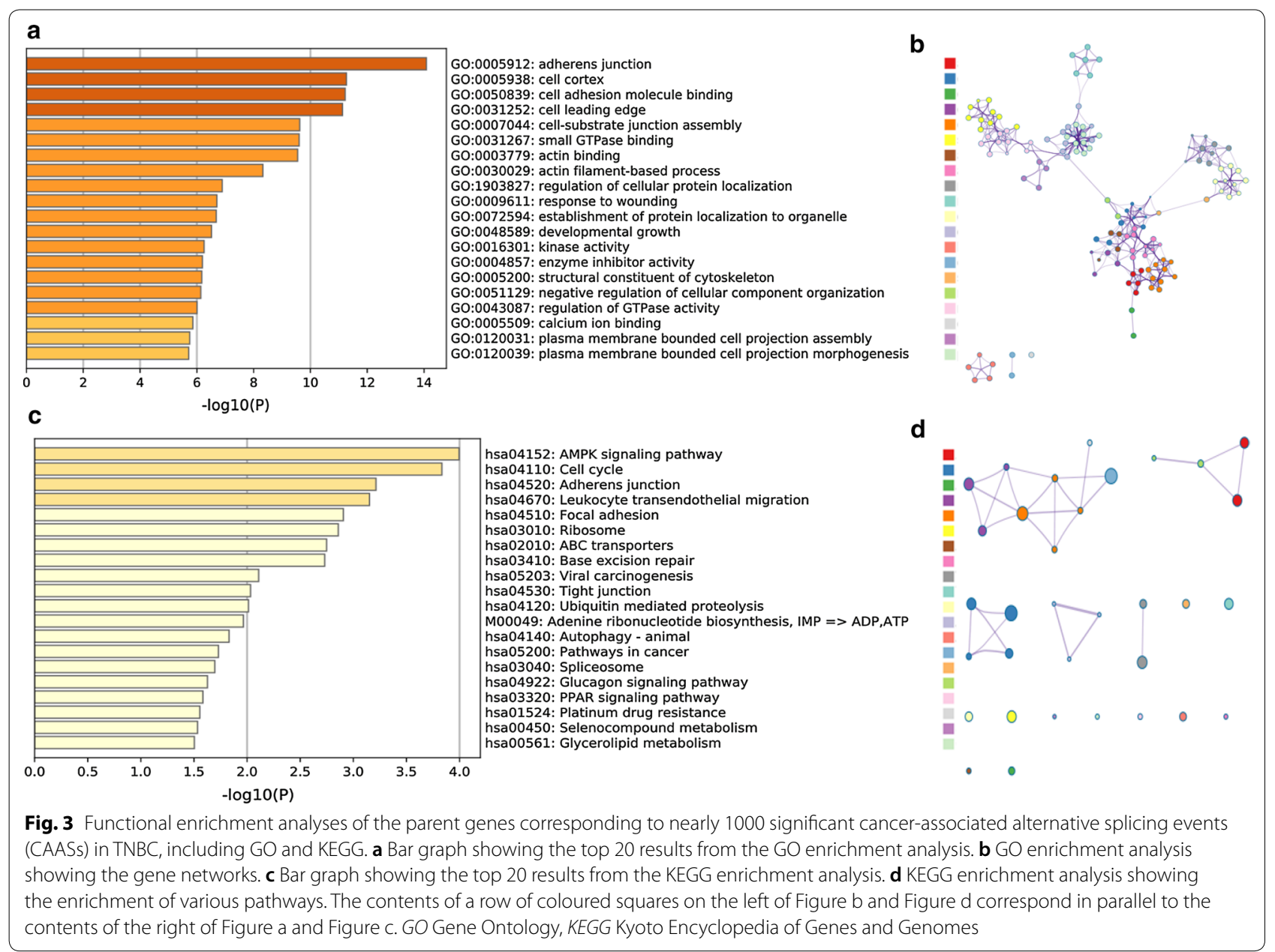

components (CC) accounted for the majority (Fig. 3a). The gene networks of the same top 20 results are displayed in Fig. 3b. Furthermore, it was suggested by KEGG enrichment analysis that some key pathways were relevant to the survival of TNBC patients, such as "MAPK signalling pathway", "Cell cycle", "Adherens junction", "Leukocyte transendothelial migration" and "Focal adhesion" (Fig. 3c), whose interrelations of various pathways are illustrated in Fig. 3d. In addition, some KEGG pathways related to TNBC tumorigenesis and treatment difficulties were abundant, including "Viral carcinogenesis", "Pathways in cancer", "Autophagy" and "Platinum drug resistance". Another interesting phenomenon was the enrichment of immune-related pathways, such as "Leukocyte transendothelial migration" and "PPAR signalling pathway", which indicated that CAAS events in TNBC are also involved in immune microenvironment formation.

\section{Association between CAAS events and the tumour microenvironment}

These findings reminded us that the tumour-immune microenvironment turbulence in TNBC could be a prognostic factor for patients. Therefore, we further performed an unsupervised consensus analysis to assess the internal profile of the immune microenvironment based on CAAS events. We divided the patients into three clusters (Fig. 4a), among which there were significance differences in the expression levels of some immune cells, such as "Mast cell resting $(P<0.001)$ ", "T cell regulatory $(P<0.01)$ ", "Macrophages M1 $(P<0.01)$ ", "Eosinophils $(P<0.01)$ ", and "T cell CD4 memory resting $(P<0.01)$ " (Fig. 4b-d). The stimulation of immune cell infiltration in the $\mathrm{C} 1$ cluster was more than that in $\mathrm{C} 2$ cluster, which indicated that the generation of different immune states may have a great relationship with the classification of CASS clusters (Fig. 4d). Furthermore, the consensus matrix heatmap showed the difference of immune cell 
a
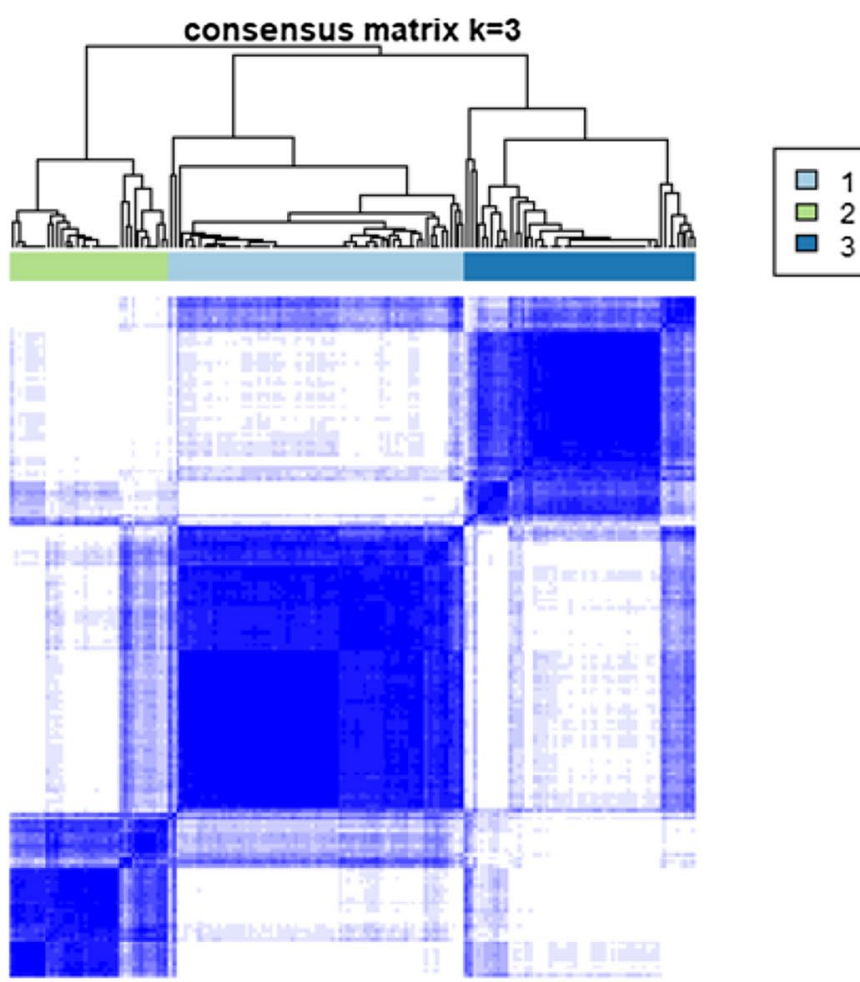

\section{b}

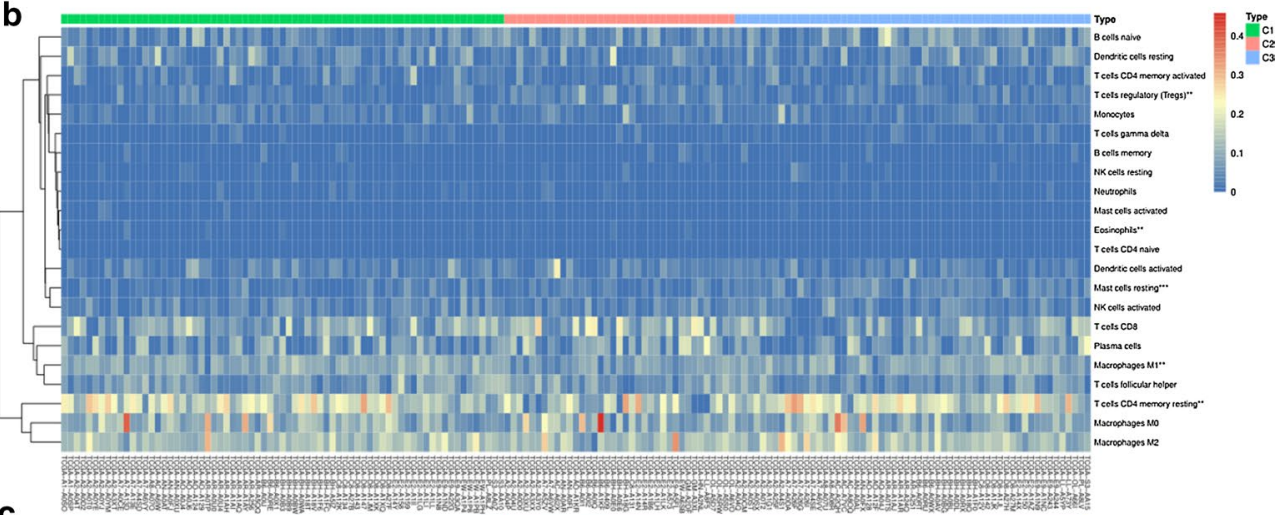

c

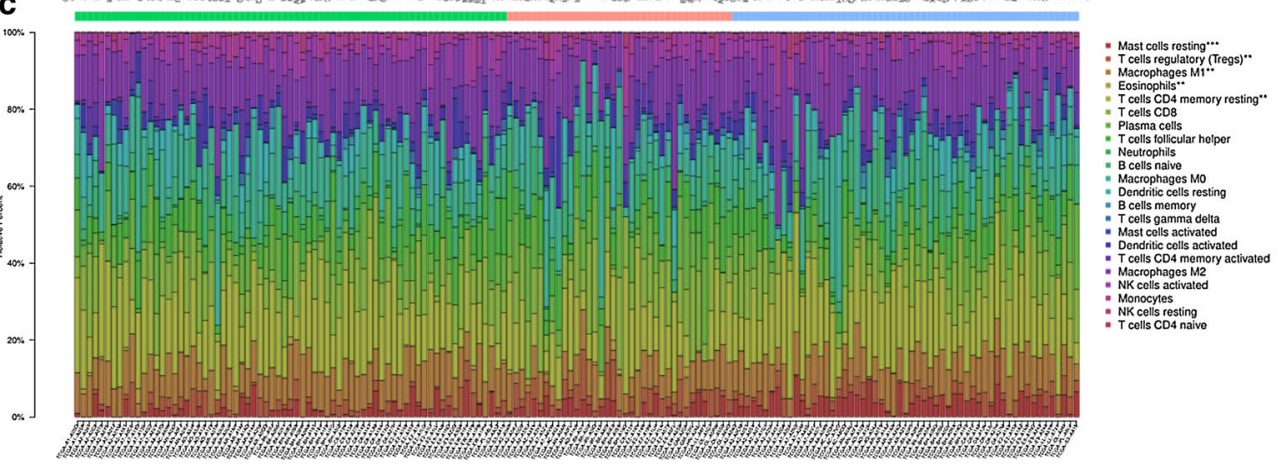

Fig. 4 CAAS-based clusters significantly associated with immune microenvironment features (CIBERSORT $P>0.05)$. a Consensus clustering analysis identification of three clusters (samples, $n=165$. The white (consensus value $=0$, samples never clustered together) and blue (consensus value $=1$, samples always clustered together) heatmap colours display sample consensus. b Heatmap of CAASs ordered by clusters showing differential expression of immune cells between patients. $\mathbf{c}$ Heatmap of CAASs ordered by clusters displaying the distribution of immune cells in each patient. The asterisk in the upper right corner of the immune cells indicates that the differential expression is significantly different. "**" represents $P<0.05$; ${ }^{\prime * * *}$ represents $P<0.01 ; " * * * "$ represents $P<0.001$. d A demonstration of the specific expression of differentially expressed immune cells in clusters according to Fig. $4 \mathrm{~b}$ and c. e Immune score and stromal score between CAAS-based clusters 

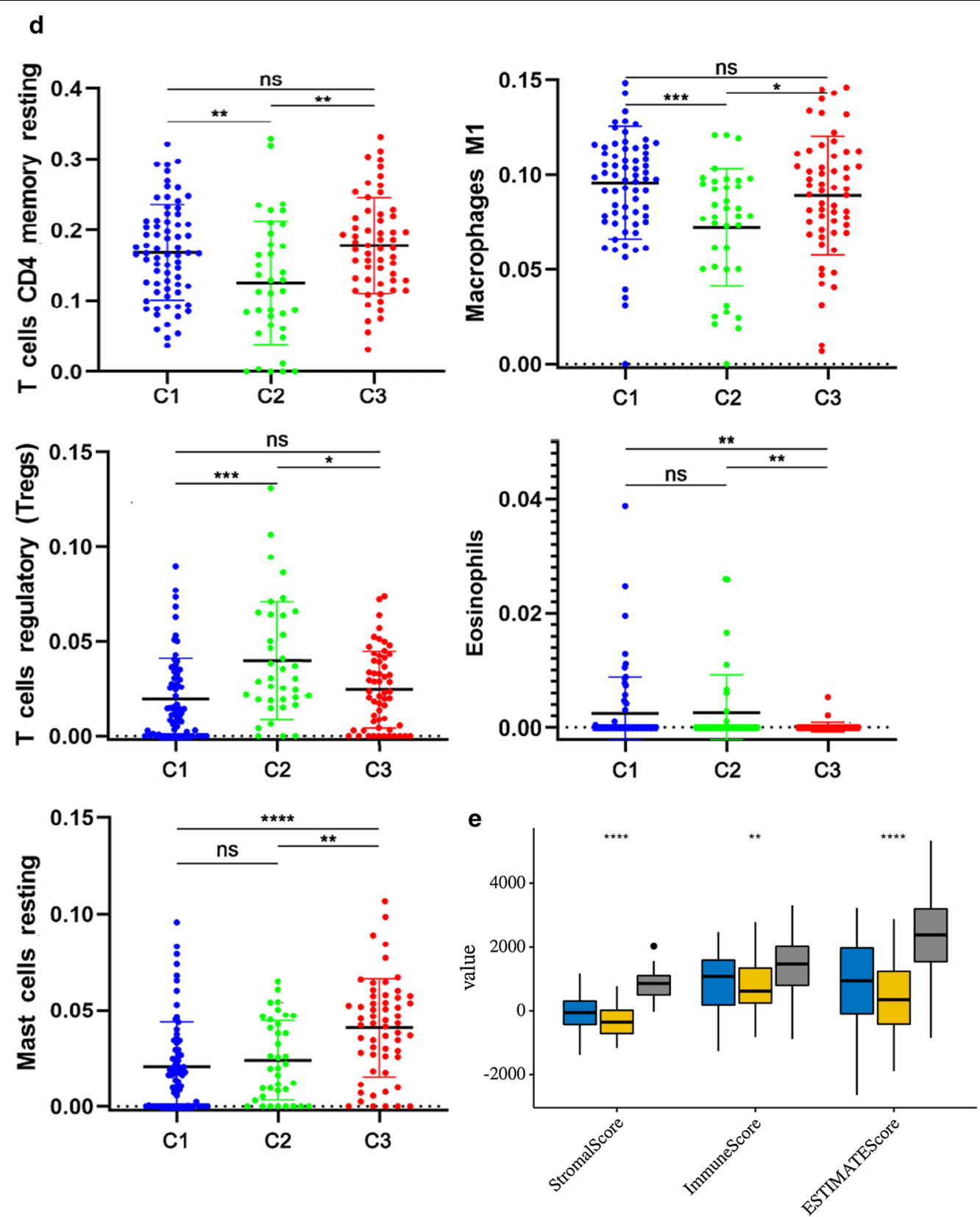

category

追 $\mathrm{c1}$

官 $\mathbf{c 2}$

追 c3

Fig. 4 continued

expression among different clusters (Fig. 4b, c). Besides, there was no difference in T cell CD4 memory resting, Macrophages M1 and T cell regulatory (adaptive immunity) between $\mathrm{C} 1$ and $\mathrm{C} 3$, but the difference was greatest in innate immune characteristics (Eosinophils and Mast cell resting) (Fig. 4d).

To further explore the underlying immunophenotypes among the three clusters, differences in the immune microenvironment among the CAAS-based clusters were further analyzed (Fig. 4E). The immune and stromal scores were calculated based on the ESTIMATE algorithm to quantify the presence of stromal cells and the infiltration of immune cells in tumor samples. We found that the immune phenotypes of the three clusters had a certain degree of heterogeneity, and we also noticed that C3 always had a higher immune and stromal score, followed by $\mathrm{C} 1$, and $\mathrm{C} 2$ was the lowest, which is similar to the situation of immune cell infiltration level (Fig. 4d). 


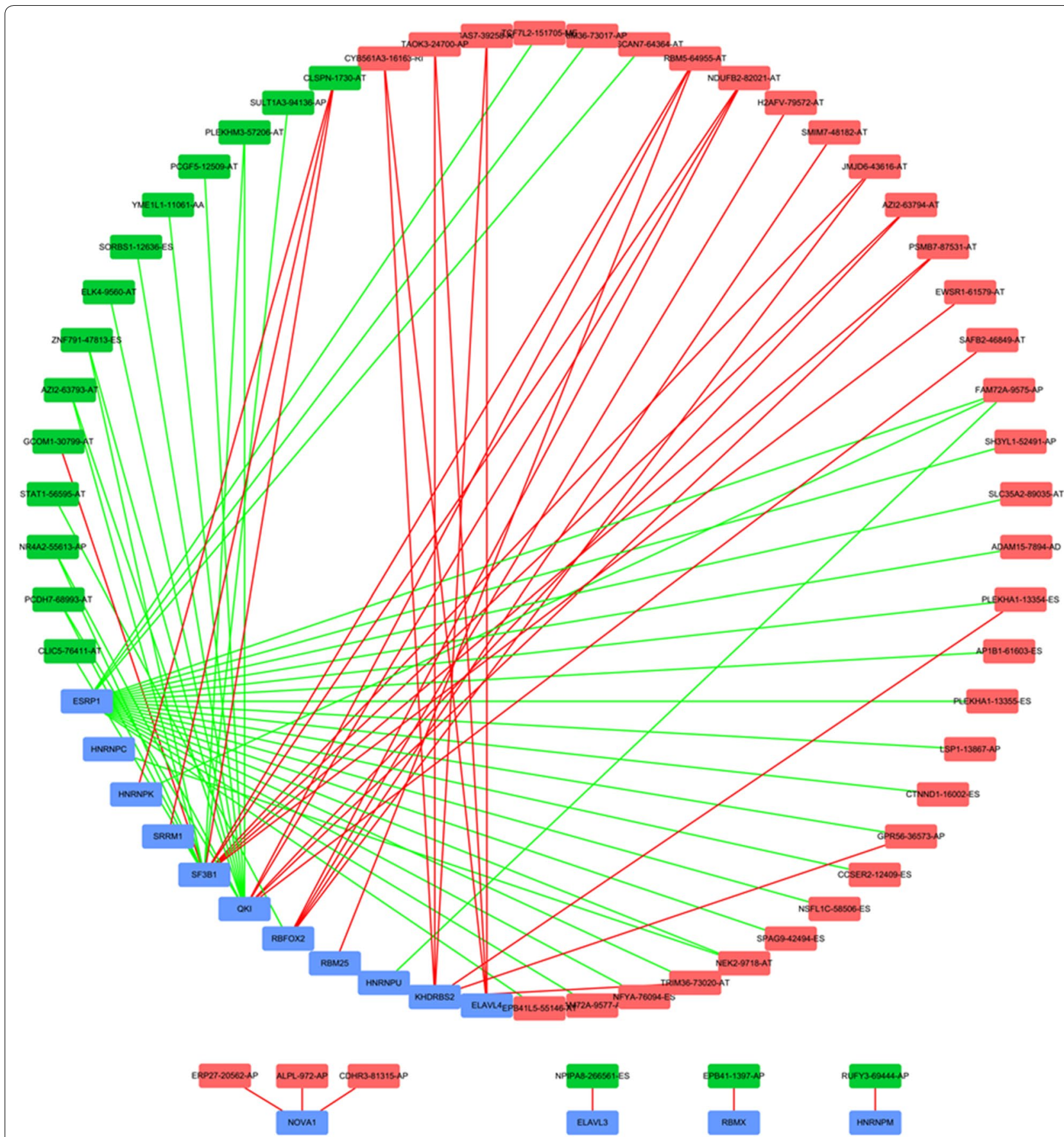

Fig. 5 Splicing correlation network in TNBC. Expression of fifteen SFsb (bule dots) were positively (red lines)/negatively (green lines) correlated with PSI values of CAAS events with favorable prognosis (green dots) or CAAS events with inferior prognosis (red dots)

\section{Regulatory network of CAAS events and SFs}

It is well known that SF plays an important regulatory role in the change and formation of AS events. For the purpose of exploring their potential connections, we downloaded a total of 71 SFs data from the SpliceAid2 database. Then Pearson correlation analysis was performed to determine the correlation between the PSI value of CAAS events and SF expression. The significant correlations $(|\mathrm{r}| \geq 0.5, p<0.001)$ were selected to construct the regulation network (Fig. 5). The regulation network consists of 53 CAAS events, of which 36 were adverse AS events (red dots) and 17 were favorable AS 


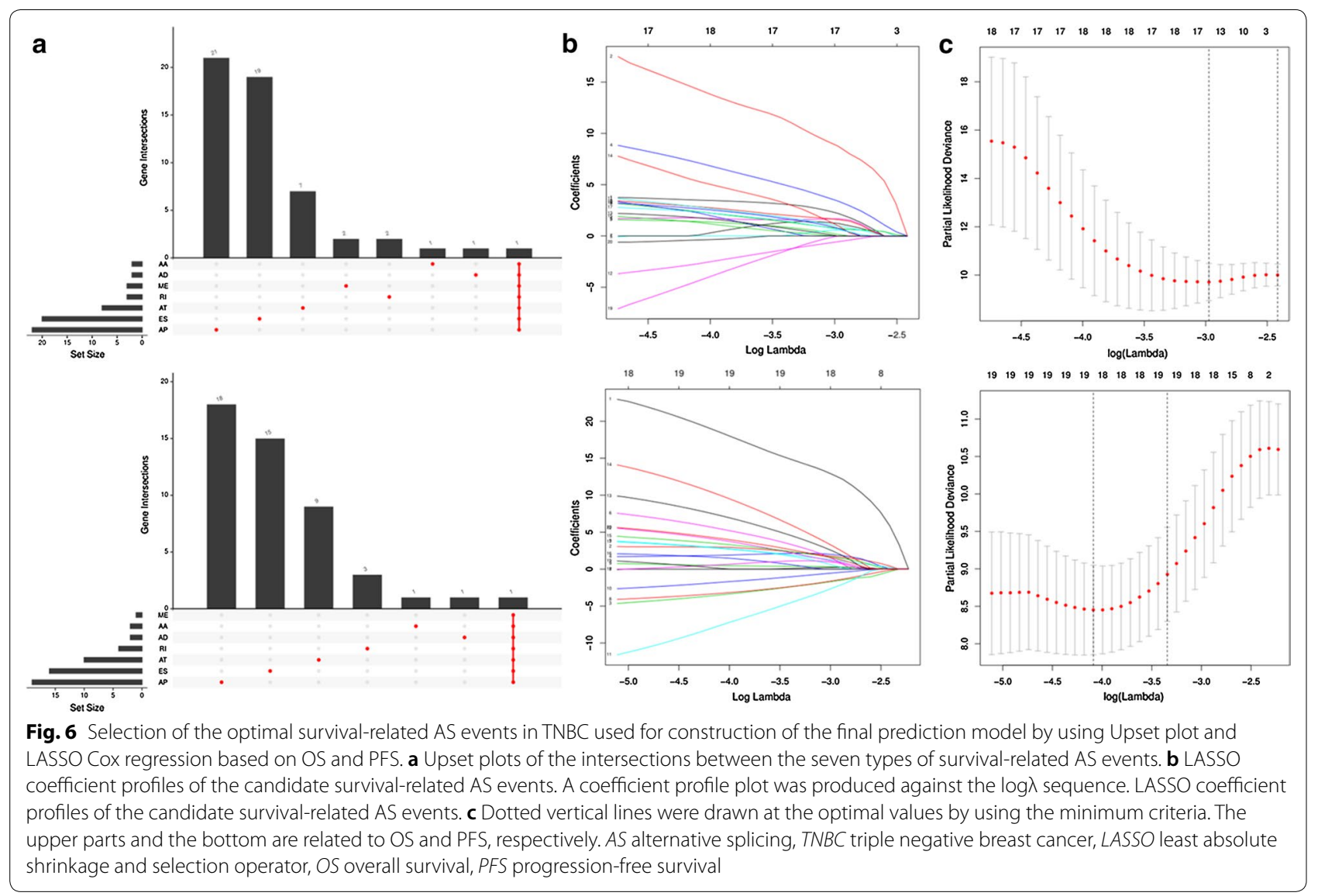

events (green dots), were significantly correlated with the $15 \mathrm{SFs}$ (blue dots). We can find that most of the SFs were correlated with multiple AS events and played opposite roles in regulating different AS events. Similarly, a part of the AS events could be regulated by different SFs. This phenomenon partly explained that the same transcript can produce multiple different splicing events. Interestingly, the majority of the adverse AS events were positively correlated with SF expression (red lines), whereas the majority of favorable AS events were negatively correlated with SF expression (green lines).

\section{Identification of survival-related CAAS events in TNBC}

By performing univariate Cox regression analysis, a total of 48 CAAS events were identified as OS-associated CAAS events, while 48 CAAS events were identified as PFS-associated CAAS events. Considering that a gene may have two or more AS events that are significantly related to the prognosis of TNBC, the UpSet plot maybe be the best choice to show the distribution of survivalrelated AS events in the seven AS types and visualize the intersection set from two study endpoints of OS and PFS (Fig. 6a; the upper and lower parts are related to OS and PFS, respectively). Both figures agreed that AP (alternate promoter) is the most common event related to the prognosis of TNBC.

\section{Establishment and assessment of the prognostic signature for TNBC patients}

After conducting univariate regression analysis, LASSO regression was performed to select the optimal survivalrelated AS events to construct the prediction models to avoid model overfitting based on OS (Additional file 1) and PFS (Additional file 2), respectively (Fig. 6b, c). Meanwhile, the risk scores of each TNBC patient were calculated, and all patients were divided into lowand high-risk groups bounded by the median risk score (Fig. 7a; the columns on the left represent OS, whereas the columns on the right represent PFS). K-M curves and log-rank tests were plotted to explore the relationship between risk score and survival status. The survival probability of low-risk patients was higher than that of high-risk patients; in other words, high-risk patients had a higher mortality rate, exactly as illustrated in Fig. 7b $(P<0.0001)$. We then applied ROC analysis to compare the predictive power of these prognostic models, which showed a robust and significantly improved performance, whose AUCs of ROC in 2, 3, and 4 years were all 


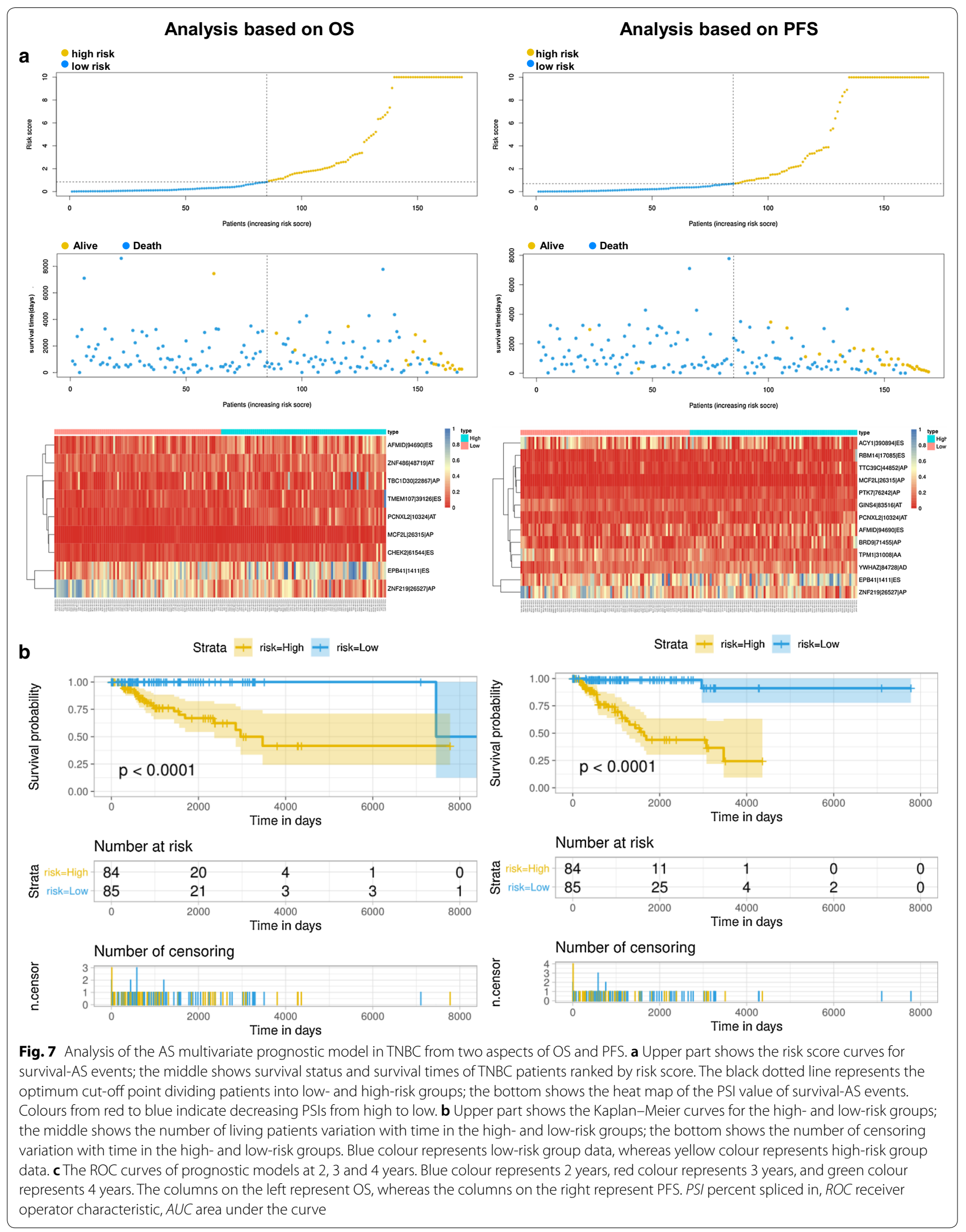



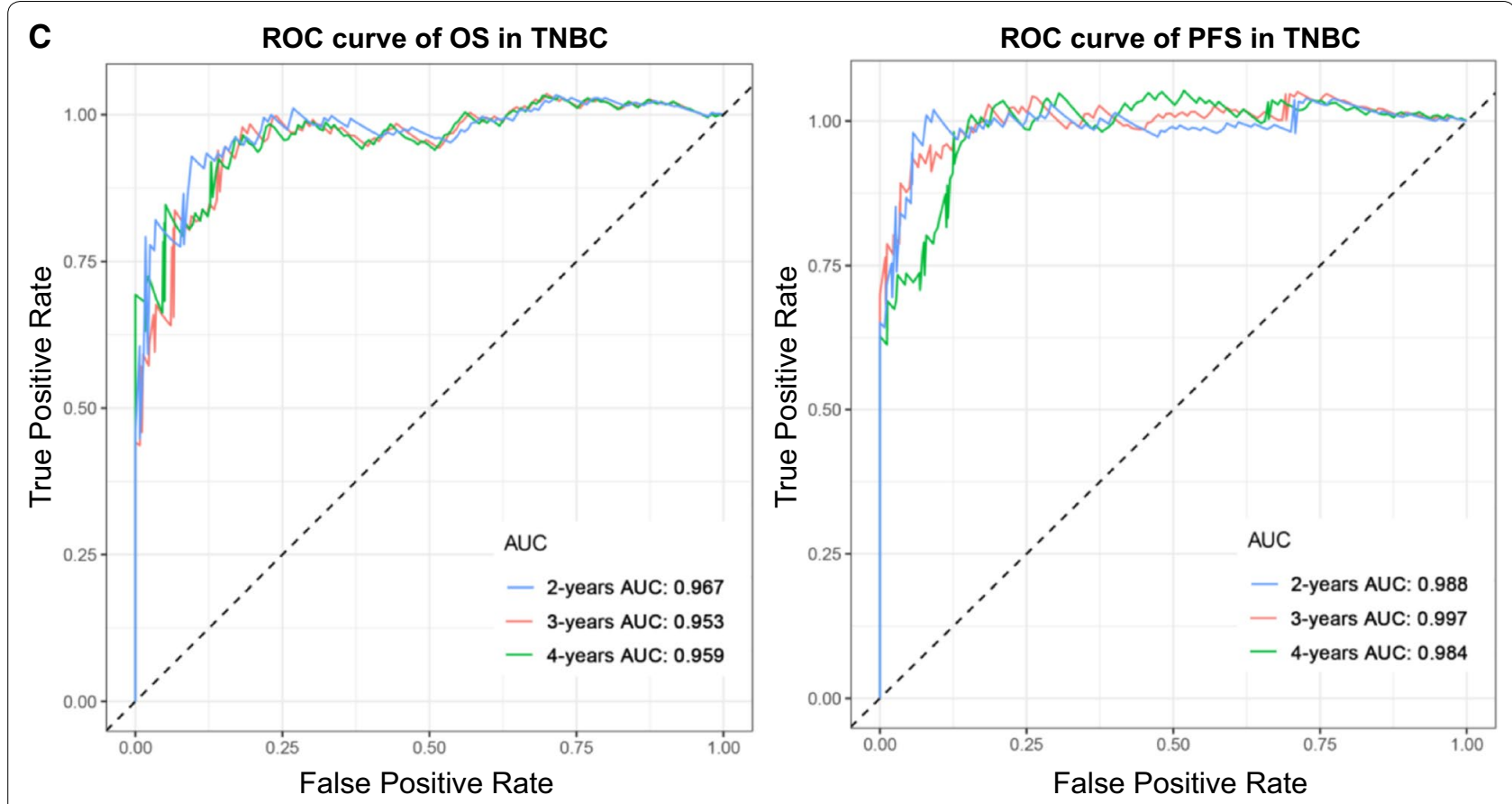

Fig. 7 continued

greater than 0.900 (Fig. 7c). Interestingly, we also found four overlapping AS events (AFMID1946901ES, MCF2L126315lAP, EPB4111411lES, ZNF219126517lAP), with significant differences found in the analysis of two different study endpoints, OS and PFS simultaneously, indicating that they are most likely independent prognostic factors (Fig. 7a).

\section{AS-clinic nomogram for predicting individual prognosis of TNBC patients}

The results of univariate Cox analysis of clinic characteristics, including OS and PFS, are displayed in Table 1, which showed that risk, AJCC, radiotherapy, and N stage were OS-related factors and that risk, AJCC, T stage, $\mathrm{N}$ stage, $M$ stage, and radiotherapy were PFS-related variables. Then, with the forward stepwise selection on optimizing AIC applied based on multivariate Cox analysis (Table 2), we finally chose three variables, including risk, AJCC and radiotherapy, for developing OS and PFS nomograms (Fig. 8a, Fig. 8e). There was good agreement between the predicted value and the actual value, which was confirmed by the calibration curve of these nomograms for the probability of survival at 2,3 , or 4 years (Fig. $8 \mathrm{~b}-\mathrm{d}$, Fig. $8 \mathrm{f}-\mathrm{h}$ ), respectively. The $\mathrm{C}$-index for the OS nomogram was 0.939 (95\% CI, 0.900-0.978), whereas the $\mathrm{C}$-index for the PFS nomogram was 0.867 (95\% CI, 0.777-0.957). These outcomes revealed that the nomogram had major clinical application value in predicting long-term survival probability.

\section{Discussion}

Recently, great breakthroughs have been made in the study of the potential significance of AS profiling in tumour biology with the enormous progress of highthroughput sequencing technology. Although the prognostic capacity of AS events has been widely confirmed in many cancers [21-24], the comprehensive profiling of AS events in TNBC patients is still lacking. In this study, we obtained and integrated clinical and AS events data from the cancer genome atlas (TCGA) and the SpliceSeq database to obtain 1194 CAAS events and identified the AS events related to survival. Moreover, two CAAS event-based signatures were generated. It was found that the prognosis of patients in the low-risk group was better than that in the high-risk group. Additionally, CAASbased risk, AJCC and radiotherapy were identified as independent OS- and PFS-related variables, which were incorporated into nomograms, and the results indicated that the two nomograms can serve as effective tools for clinical practice with TNBC patients.

In the present study, two CAAS event-based signatures were developed and showed a favourable predictive capacity. Interestingly, five overlapping AS events were identified among the splicing events, with significant differences based on OS and PFS simultaneously, including 
Table 1 Screening clinical variables related to prognosis by univariate analysis in TNBC cohort

\begin{tabular}{|c|c|c|c|c|c|c|c|c|}
\hline \multirow[b]{3}{*}{ Risk } & \multicolumn{4}{|l|}{ os } & \multicolumn{4}{|l|}{ PFS } \\
\hline & \multirow{2}{*}{$\begin{array}{l}\text { HR } \\
24.311\end{array}$} & \multicolumn{2}{|l|}{$95 \% \mathrm{Cl}$} & \multirow{2}{*}{$\begin{array}{l}\boldsymbol{P} \\
0.002\end{array}$} & \multirow{2}{*}{$\begin{array}{l}\text { HR } \\
20.068\end{array}$} & \multicolumn{2}{|c|}{$95 \% \mathrm{Cl}$} & \multirow{2}{*}{$\frac{P}{0.000}$} \\
\hline & & 3.264 & 181.084 & & & 4.771 & 84.404 & \\
\hline Age & 1.018 & 0.985 & 1.052 & 0.288 & 1.006 & 0.978 & 1.034 & 0.699 \\
\hline \multicolumn{9}{|l|}{ AJCC } \\
\hline$|-| \mid$ & & & & 0.000 & & & & 0.000 \\
\hline III-IV & 6.079 & 2.492 & 14.829 & 0.000 & 4.879 & 2.259 & 10.535 & 0.000 \\
\hline NA & 1.203 & 0.138 & 10.462 & 0.867 & 1.300 & 0.171 & 9.866 & 0.800 \\
\hline \multicolumn{9}{|l|}{ M } \\
\hline 0 & & & & 0.323 & & & & 0.001 \\
\hline 1 & 3.478 & 0.677 & 17.864 & 0.135 & 18.603 & 3.882 & 89.139 & 0.000 \\
\hline NA & 1.223 & 0.280 & 5.339 & 0.789 & 1.219 & 0.364 & 4.084 & 0.748 \\
\hline $\mathrm{N}$ & 4.468 & 1.746 & 11.430 & 0.002 & 2.994 & 1.423 & 6.296 & 0.004 \\
\hline \multicolumn{9}{|l|}{$\mathrm{T}$} \\
\hline $1-2$ & & & & 0.190 & & & & 0.131 \\
\hline $3-4$ & 2.574 & 0.931 & 7.116 & 0.068 & 2.425 & 1.026 & 5.735 & 0.044 \\
\hline NA & 0.000 & 0.000 & 0.000 & 0.981 & 0.000 & 0.000 & 0.000 & 0.983 \\
\hline \multicolumn{9}{|c|}{ Radiotherapy } \\
\hline No & & & & 0.000 & & & & 0.001 \\
\hline Yes & 0.315 & 0.121 & 0.826 & 0.019 & 0.439 & 0.173 & 1.111 & 0.082 \\
\hline NA & 0.060 & 0.017 & 0.207 & 0.000 & 0.159 & 0.060 & 0.419 & 0.000 \\
\hline \multicolumn{9}{|l|}{ Race } \\
\hline Asian & & & & 0.304 & & & & 0.791 \\
\hline White & 0.192 & 0.023 & 1.571 & 0.124 & 0.363 & 0.046 & 2.834 & 0.334 \\
\hline Black & 0.361 & 0.044 & 2.973 & 0.344 & 0.435 & 0.054 & 3.521 & 0.435 \\
\hline NA & 0.000 & 0.000 & 0.000 & 0.985 & 0.000 & 0.000 & 0.000 & 0.980 \\
\hline
\end{tabular}

HR hazard ratio, AJCC American Joint Committee on Cancer, NA not applicable

Table 2 Detailed information of specific AS events involved in final prognostic model by multivariate analysis

\begin{tabular}{|c|c|c|c|c|c|c|c|c|}
\hline \multirow[b]{3}{*}{ Risk } & \multicolumn{4}{|l|}{ os } & \multicolumn{4}{|l|}{ PFS } \\
\hline & \multirow{2}{*}{$\begin{array}{l}\text { HR } \\
32.503\end{array}$} & \multicolumn{2}{|c|}{$95 \% \mathrm{Cl}$} & \multirow{2}{*}{$\begin{array}{l}P \\
0.013\end{array}$} & \multirow{2}{*}{$\begin{array}{l}\text { HR } \\
0.040\end{array}$} & \multicolumn{2}{|c|}{$95 \% \mathrm{Cl}$} & \multirow{2}{*}{$\frac{P}{0.002}$} \\
\hline & & 2.105 & 501.818 & & & 0.005 & 0.301 & \\
\hline \multicolumn{9}{|l|}{ AJCC } \\
\hline$|-| \mid$ & & & & 0.000 & & & & 0.000 \\
\hline III-IV & 16.561 & 4.728 & 58.007 & 0.000 & 6.939 & 2.786 & 17.282 & 0.000 \\
\hline NA & 0.273 & 0.026 & 2.876 & 0.280 & 11.715 & 0.674 & 203.462 & 0.091 \\
\hline \multicolumn{9}{|c|}{ Radiotherapy } \\
\hline No & & & & 0.000 & & & & 0.002 \\
\hline Yes & 0.318 & 0.102 & 0.995 & 0.049 & 0.543 & 0.198 & 1.490 & 0.236 \\
\hline NA & 0.023 & 0.005 & 0.113 & 0.000 & 0.164 & 0.053 & 0.504 & 0.002 \\
\hline
\end{tabular}

HR hazard ratio, AJCC American Joint Committee on Cancer, NA not applicable

AFMIDl946901ES, MCF2Ll26315lAP, EPB4111411lES, ZNF2191265171AP and PCNXL2|10324|AT, suggesting that they are the most likely independent prognostic factors. AFMID (arylformamidase) is located on chromosome 17q25.3 and encodes arylformamidase, a control enzyme in tryptophan metabolism. Consistent with our findings, Krainer et al. also found that specific splicing events of the AFMID gene are significantly associated with survival in hepatocellular carcinoma (HCC) patients. The conversion of the AFMID subtype 
(See figure on next page.)

Fig. 8 AS-clinic nomograms for predicting the individualized survival rates of TNBC patients for two aspects of OS and PFS. a, e Construction of AS-clinic nomograms for TNBC patients to predict 2, 3, and 4 year OS (a) and PFS (e), which were incorporated with 3 variables, including risk, AJCC and radiotherapy. $\mathbf{b}-\mathbf{d}, \mathbf{f}-\mathbf{h}$ Calibration plots of the AS-clinic nomograms in terms of agreement between nomogram-predicted and observed 2 , 3 , and 4 year outcomes of the TNBC cohort, including OS (b-d) and PFS (f-h). The $45^{\circ}$ dashed line represents the ideal performance. The actual performances of the model are represented by the red lines, and the figures from left to right show the 2,3 , and 4 year results

represents a new regulatory step in tryptophan/kynurenine metabolism, revealing the disruption of neonatal NAD + biosynthesis in hepatocytes in the early stages of tumour development [32]. In addition, NAD + is an important coenzyme in the energy metabolism of eukaryotic cells [33]. Previous studies have shown that $\mathrm{NAD}+$ supplementation can extend the life span of mice [34]. However, the ratio of NAD + /NADH in cancer cells is very low, and they maintain enough NAD + by converting pyruvate to lactate to achieve high-speed glycolysis while shutting down other sources of NAD + production [35]. In summary, repairing AFMID splices may lead to the augmentation of NAD + production and DNA repair. Therefore, it is expected that AFMID splicing could become a therapeutic target and a source of new cancer drugs after additional research.

Regarding PCNXL2 (pecanex-like 2), namely FLJ11383|KIAA0435, a previous report suggested that it might have a certain influence on the tumorigenesis of colorectal carcinomas with high microsatellite instability [36], which may be a new breakthrough point for immunotherapy with the progress of genomics research and the maturation of protein antibody preparation technology. Nevertheless, the main focuses of recent studies were established based on the correlation of PCNXL2 as a novel susceptibility locus of thyroid cancer [37, 38], which was speculated to be related to the prognosis of thyroid cancer. In addition, there is a broad consensus that the emergence of chemotherapy resistance is also a major problem affecting the therapeutic effect. MC2L is one of the guanine nucleotide exchange factors, which may link the potential signalling pathway through RAC1, RHOA and CDC42, also named DBS/DBLs Big Sister, belonging to the DBL family [39]. Research has shown that MCF2L/DBS MCF2L may play an important role in gemcitabine resistance of primary pancreatic cancer patients [40], which may provide some explanations for the causes of TNBC chemotherapy resistance. It should be noted that the EPB41 gene encodes Erythrocyte Membrane Protein Band 4.1, which belongs to the family of cytoskeletal proteins that play important roles in maintaining normal cell morphology and cell adhesion, migration, division, and intercellular signalling [41-43]. Many studies have revealed the capacity of EPB41 to predict the prognosis of various cancers and its critical role in the development of tumours, such as breast cancer [44], meningiomas [45], prostate cancer [46], and hepatocellular carcinoma [47]. Together, this evidence supports the biological relevance of EPB41 in tumour biology. Our findings are mostly consistent with the above results, but further research is still needed.

Moreover, we also found that the parent genes of CAAS events were significantly enriched in several functions and pathways, revealing the potential molecular functions and signalling pathways associated with TNBC progression and treatment difficulties. In addition, some immune-related mechanisms were also identified, such as "Leukocyte transendothelial migration" and "PPAR signalling pathway". Previous research had proven that PPAR $\gamma$ (peroxisome proliferator-activated receptors) is a key regulator of lipid and glucose metabolism in many cell types, with robust anti-inflammatory activity in immune cells $[48,49]$. Moreover, $\mathrm{PPAR}^{\mathrm{High}} / \mathrm{RXR}^{\mathrm{S} 427 \mathrm{~F} / \mathrm{Y}}$ interferes with $\mathrm{CD} 8+\mathrm{T}$ cell infiltration and participates in immunotherapy resistance. Knockdown of PPARy or RXR $\alpha$ and inhibition of PPAR can restore immune surveillance and sensitivity to immunotherapy [50]. Inspired by these findings, we further investigated potential interactions between CAAS events and the tumour microenvironment. Unsupervised clustering analysis was adopted and found that the expression levels of some immune cells between clusters, such as "Mast cell resting $(P<0.001)$ ", "T cell regulatory $(P<0.01)$ ", "Macrophages M1 $(P<0.01)$ ", "Eosinophils $(P<0.01)$ ", and "T cell CD4 memory resting $(P<0.01)$ ", were significantly different, demonstrating that differences in CAAS could lead to changes in the tumour immune microenvironment. Previous studies have shown that high infiltration of M1 [51] and CD4 T cells, E0 expression [52], mast cell expression [53], and low Treg expression [54] or other states are the best state of hot tumours, which can increase the efficacy of immunotherapy. What's more, the main difference between the three clusters was the infiltration level of innate and acquired immune cells, which was verified by differential analysis of the underlying immunophenotype and immune microenvironment.In addition, we also discovered the heterogeneity of the immune microenvironment in TNBC based on the consensus matrix heatmap, which could to some extent explain the clinical phenomenon that PD-1 / PD-L1 immunotherapy had different effects on TNBC patients. 
a

$\begin{array}{llllll}0 & 1 & 2 & 3 & 4 & 5\end{array}$

Points

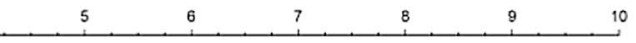

Risk

AJCC

Radiatherapy

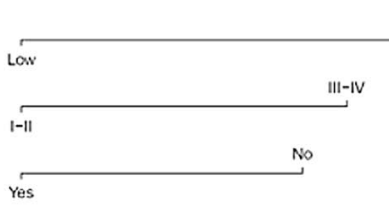

Total Points

2 year OS Probability

3 year OS Probability

4 year OS Probability
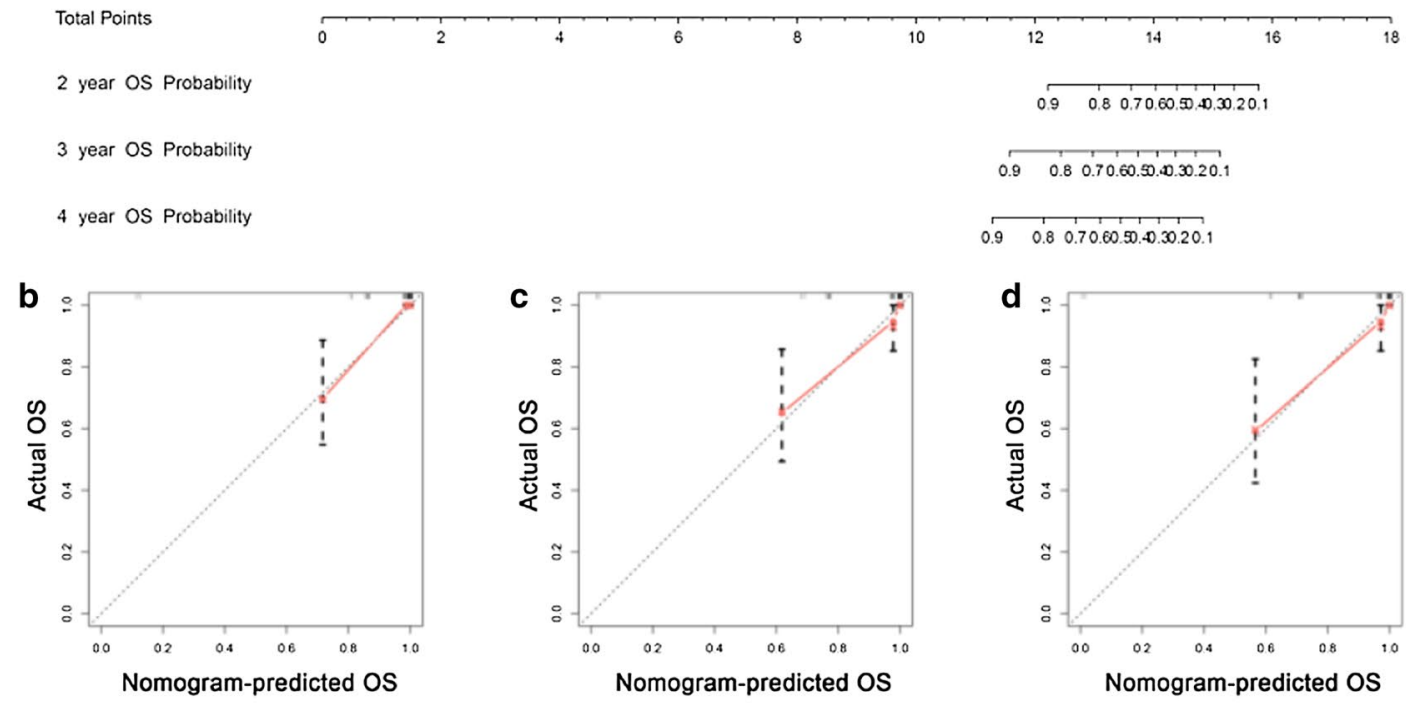

$$
\begin{array}{lll}
0.9 & 0.8 & 0.70 .60 .50 .40 .30 .20 .1
\end{array}
$$

$0.9 \quad 0.8 \quad 0.70 .60 .50 \cdot 0.30 .20 .1$

$\begin{array}{lll}0.9 \quad 0.8 & 0.70 .60 .90 .40 .3020 .1\end{array}$

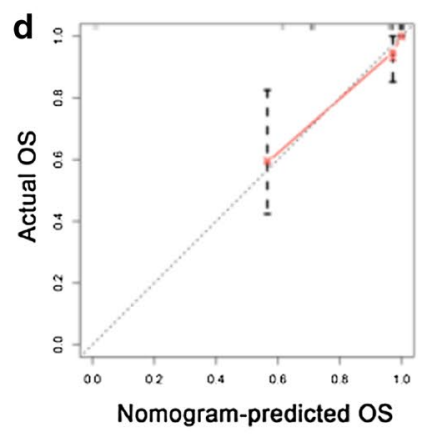

e Points

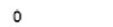

2

3

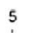

7

8

9 10

Risk

III-IV

AJCC

Radiatherapy

Low

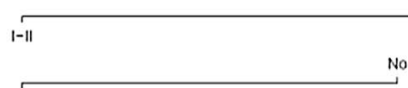

Total Points

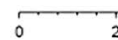

No

2 year PFS Probability

3 year PFS Probability

4 year PFS Probability
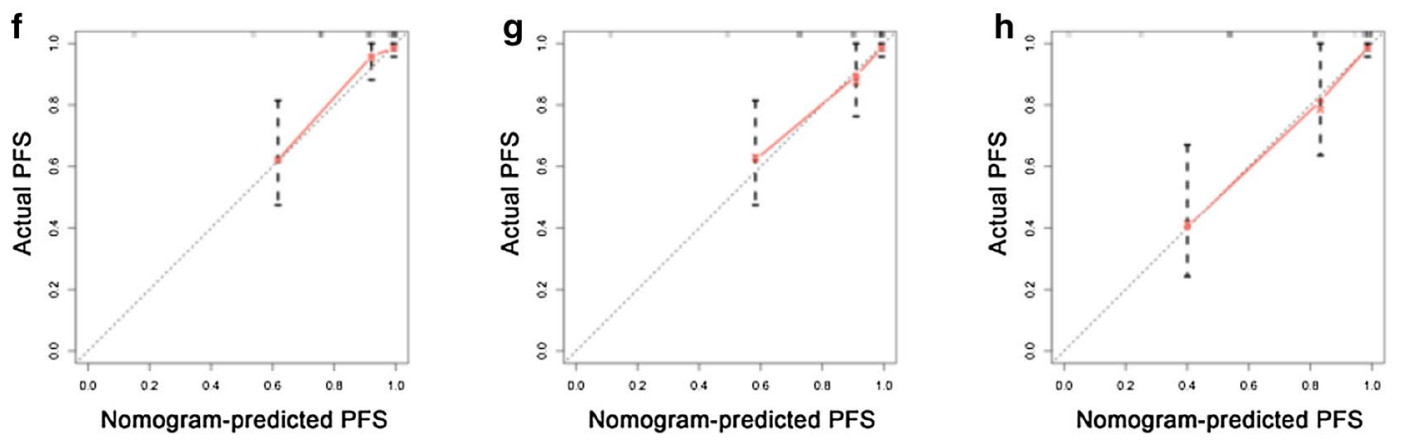
Additionally, the potential involvement of splicing factors was also considered in our analysis. Splicing correlation network revealed that the relationship between SFs and AS was not a one-to-one correspondence, but had multiple interactions, which partly explained the diversity of AS events. And the other thing that was interesting was that the majority of the adverse AS events were positively correlated with SF expression, whereas the majority of favorable AS events were negatively correlated with SF expression, which may provide a way to elucidate the potential mechanism of splicing pathways involved in patient survival. However, due to the limited number of acquired SFs, we were unable to screen out survivalrelated data for study. Therefore, the survival regulation mechanism of the SF-AS network was not yet clear and in-depth functional investigation was necessary.

Our study was the first to analyse the AS profile in TNBC from multiple perspectives, selected the most relevant prognostic factors, established a prognostic model with high accuracy, and further accurately predicted the individual survival rate of TNBC patients. Moreover, the immune microenvironment turbulence in the TNBC population was revealed from the perspective of immunology. However, it is inevitable that our research still has several limitations that we should consider. First, the TNBC-related data we obtained through the TCGA database included only 169 cases, the sample size was not large enough, and our results were only verified by the TCGA dataset without additional external datasets. Second, adjacent normal tissues do not necessarily represent the origin of tumour cells, whose changes in expression may not be a requirement for splicing variants to function [55]. In addition, our study is based on pure bioinformatics analysis and lacks clinical validation. Therefore, further studies of the biological role and molecular mechanism of AS events in TNBC tumorigenesis are needed.

In summary, this study has enriched AS profiling research on breast cancer, simultaneously and for the first time unveiling the characteristics of AS events in TNBC. In addition, we established a powerful prognostic model to accurately predict the prognosis of TNBC patients. More importantly, this comprehensive analysis based on differentially expressed AS events has enhanced our understanding of AS events promoting tumorigenesis and development, which are more likely to be potential clinical biomarkers and therapeutic targets and provide guiding significance for future basic research and clinical work.

\section{Conclusion}

Based on the comprehensive bioinformatics analysis, our study was the first to showed that AS events were closely linked to tumorigenesis and the immune microenvironment in TNBC. Further, we established the prediction model with good performance in basis of survival-related AS events. Besides, we identified five independent prognostic factors, including AFMIDl94690lES, MCF2L126315lAP, EPB4111411lES, ZNF219l26517lAP and PCNXL2|10324|AT, each worthy of further investigation.These findings provided insight into the connection between AS events and TNBC.

\section{Supplementary information}

Supplementary information accompanies this paper at https://doi. org/10.1186/s12967-020-02454-1.

Additional file 1: The optimal OS-related AS events was selected by LASSO regression for constructing the prediction models.

Additional file 2: The optimal PFS-related AS events was selected by LASSO regression for constructing the prediction models.

\section{Acknowledgements}

None.

\section{Authors' contributions}

WFL conceived and designed the study with $\mathrm{CH}$ and SSY. SSY and HC drafted the manuscript and analysed the data with $L X L$ and LYC. XDD, QJY, FL,DJZ and $Y Z$ formatted the images and the article. $C Z$ and $X L$ reviewed the data. All authors read and approved the final manuscript.

\section{Funding}

This work was supported by the National Natural Science Foundation of China (No. 80218103).

\section{Availability of data and materials}

The datasets generated during and/or analysed during the current study are available from the corresponding author on reasonable request.

Ethics approval and consent to participate

There was no enthics associated with this work because the study didin't involve animal experiments or human specimens.

\section{Consent for publication}

Not applicable.

\section{Competing interests}

The authors have no conflicts of interest or disclosures relevant to this work.

\section{Author details}

${ }^{1}$ Department of Chemoradiation Oncology, The First Affiliated Hospital of Wenzhou Medical University, 2 Fuxue Road, Wenzhou, Zhejiang 325000 , People's Republic of China. ${ }^{2}$ Department of Orthopaedic Surgery, the Affiliated Hospital of Qingdao University, Qingdao 266071, China. ${ }^{3}$ Department of Obstetrics and Gynecology, The First Affiliated Hospital of Wenzhou Medical University, Wenzhou, Zhejiang, People's Republic of China. ${ }^{4}$ Department of Dermatology, The First Affiliated Hospital of Wenzhou Medical University, Wenzhou, Zhejiang, People's Republic of China.

Received: 4 April 2020 Accepted: 21 July 2020

Published online: 28 July 2020

References

1. Siegel RL, Miller KD, Jemal A. Cancer statistics, 2019. CA Cancer J Clin. 2019:69:7-34

2. Anders CCL. Understanding and treating triple-negative breast cancer. Oncology (Williston Park). 2008;22:1233-9. 
3. Sheikh A, Hussain SA, Ghori Q, Naeem N, Fazil A, Giri S, Sathian B, Mainali $P$, Al Tamimi DM. The spectrum of genetic mutations in breast cancer. Asian Pac J Cancer Prev. 2015;16:2177-85.

4. Corbex M, Bouzbid S, Traverse-Glehen A, Aouras H, McKay-Chopin S, Carreira C, Lankar A, Tommasino M, Gheit T. Prevalence of papillomaviruses, polyomaviruses, and herpesviruses in triple-negative and inflammatory breast tumors from algeria compared with other types of breast cancer tumors. PLOS ONE. 2014;9:e114559.

5. Adams SSP, Rugo HS, Winer EP, Loirat D, Awada A, Cescon DW, Iwata H, Campone M, Nanda R, et al. Pembrolizumab monotherapy for previously treated metastatic triple-negative breast cancer: cohort A of the phase 2 KEYNOTE-086 study. Ann Oncol. 2019;30:397-404.

6. Schmid P, Adams S, Rugo HS, Schneeweiss A, Barrios CH, Iwata H, Dieras V, Hegg R, Im SA, Shaw Wright G, et al. Atezolizumab and nab-paclitaxel in advanced triple-negative breast cancer. N Engl J Med. 2018;379:2108-21.

7. Adams SLS, Toppmeyer D, Cescon DW, De Laurentiis M, Nanda R, Winer EP, Mukai H, Tamura K, Armstrong A, et al. Pembrolizumab monotherapy for previously untreated, PD-L1-positive, metastatic triple-negative breast cancer: cohort B of the phase II KEYNOTE-086 study. Ann Oncol. 2019:30:405-11.

8. Yao H, He G, Yan S, Chen C, Song L, Rosol TJ, Deng X 1. Triple-negative breast cancer: is there a treatment on the horizon? Oncotarget. 2017:8:1913-24

9. Pogoda K, Niwinska A, Murawska M, Pienkowski T. Analysis of pattern, time and risk factors influencing recurrence in triple-negative breast cancer patients. Med Oncol. 2013;30:388.

10. Echeverria GV, Powell E, Seth S, Ge Z, Carugo A, Bristow C, Peoples M, Robinson F, Qiu H, Shao J, et al. High-resolution clonal mapping of multi-organ metastasis in triple negative breast cancer. Nat Commun. 2018;9:5079.

11. Li Z-X, Zheng Z-Q, Wei Z-H, Zhang L-L, Li F, Lin L, Liu R-Q, Huang X-D, Lv J-W, Chen F-P, et al. Comprehensive characterization of the alternative splicing landscape in head and neck squamous cell carcinoma reveals novel events associated with tumorigenesis and the immune microenvironment. Theranostics. 2019;9:7648-65.

12. Zhang S, Wu X, Diao P, Wang C, Wang D, Li S, Wang Y, Cheng J. Identification of a prognostic alternative splicing signature in oral squamous cell carcinoma. J Cell Physiol. 2020;235:4804-13.

13. Montes M, Sanford BL, Comiskey DF, Chandler DS. RNA splicing and sisease: animal models to therapies. Trends Genet. 2019;35:68-87.

14. Kelemen O, Convertini P, Zhang Z, Wen Y, Shen M, Falaleeva M, Stamm S. Function of alternative splicing. Gene. 2013;514:1-30

15. Nilsen TW, Graveley BR. Expansion of the eukaryotic proteome by alternative splicing. Nature. 2010;463:457-63.

16. Chen L, Kostadima M, Martens JHA, Canu G, Garcia SP, Turro E, Downes K, Macaulay IC, Bielczyk-Maczynska E, Coe S, et al. Transcriptional diversity during lineage commitment of human blood progenitors. Science. 2014;345:1251033.

17. Sultana N, Dienes B, Benedetti A, Tuluc P, Szentesi P, Sztretye M, Rainer J, Hess MW, Schwarzer C, Obermair GJ, et al. Restricting calcium currents is required for correct fiber type specification in skeletal muscle. Development. 2016;143:1547-59.

18. Zong Z, Li H, Yi C, Ying H, Zhu Z, Wang H. Genome-wide profiling of prognostic alternative splicing signature in colorectal cancer. Front Oncol. 2018;8:537.

19. Oltean S, Bates DO. Hallmarks of alternative splicing in cancer. Oncogene. 2014;33:5311-8

20. Koh CM, Bezzi M, Low DH, Ang WX, Teo SX, Gay FP, Al-Haddawi M, Tan SY, Osato M, Sabo A, et al. MYC regulates the core pre-mRNA splicing machinery as an essential step in lymphomagenesis. Nature. 2015;523:96-100.

21. Wu F, Chen Q, Liu C, Duan X, Hu J, Liu J, Cao H, Li W, Li H. Profiles of prognostic alternative splicing signature in hepatocellular carcinoma. Cancer Med. 2020;9:2171-80.

22. Liang Y, Song J, He D, Xia Y, Wu Y, Yin X, Liu J. Systematic analysis of survival-associated alternative splicing signatures uncovers prognostic predictors for head and neck cancer. J Cell Physiol. 2019;234(9):15836-46.

23. Meng T, Huang R, Zeng Z, Huang Z, Yin H, Jiao C, Yan P, Hu P, Zhu X, Li $Z$, et al. Identification of prognostic and metastatic alternative splicing signatures in kidney renal clear cell carcinoma. Front Bioeng Biotechnol. 2019;7:270.
24. Yang C, Wu Q, Huang K, Wang X, Yu T, Liao X, Huang J, Zhu G, Gong Y, Han C, et al. Genome-wide profiling reveals the landscape of prognostic alternative splicing signatures in pancreatic ductal adenocarcinoma. Front Oncol. 2019;9:511.

25. Ryan MC, Cleland J, Kim R, Wong WC, Weinstein JN. SpliceSeq: a resource for analysis and visualization of RNA-Seq data on alternative splicing and its functional impacts. Bioinformatics. 2012;28:2385-7.

26. Giulietti M, Piva F, D'Antonio M, De D'Onorio P, Paoletti D, Castrignano T, D'Erchia AM, Picardi E, Zambelli F, Principato G, et al. SpliceAid-F: a database of human splicing factors and their RNA-binding sites. Nucleic Acids Res. 2013;41:D125-131.

27. Sveen A, Kilpinen S, Ruusulehto A, Lothe RA, Skotheim RI. Aberrant RNA splicing in cancer; expression changes and driver mutations of splicing factor genes. Oncogene. 2016;35:2413-27.

28. Dvinge H, Kim E, Abdel-Wahab O, Bradley RK. RNA splicing factors as oncoproteins and tumour suppressors. Nat Rev Cancer. 2016;16:413-30.

29. Lex A, Gehlenborg N, Strobelt H, Vuillemot R, Pfister H. UpSet: visualization of intersecting sets. IEEE Trans Vis Comput Graph. 2014;20:1983-92.

30. Conway JR, Lex A, Gehlenborg N. UpSetR: an R package for the visualization of intersecting sets and their properties. Bioinformatics. 2017;33:2938-40.

31. Gao J, Kwan PW, Shi D. Sparse kernel learning with LASSO and Bayesian inference algorithm. Neural Netw. 2010;23:257-64.

32. Lin KT, Ma WK, Scharner J, Liu YR, Krainer AR. A human-specific switch of alternatively spliced AFMID isoforms contributes to TP53 mutations and tumor recurrence in hepatocellular carcinoma. Genome Res. 2018;28(3):275-84.

33. Canto C, Menzies KJ, Auwerx J. NAD(+) metabolism and the control of energy homeostasis: a balancing act between mitochondria and the nucleus. Cell Metab. 2015;22:31-533.

34. Zhang HRD, Wu Y, Gariani K, Wang X, Luan P, D'Amico D, Ropelle ER, Lutolf MP, Aebersold R, et al. NAD+ repletion improves mitochondrial and stem cell function and enhances life span in mice. Science. 2016;352:1436-43.

35. Vander Heiden MG, DeBerardinis RJ. Understanding the intersections between metabolism and cancer biology. Cell. 2017;168:657-69.

36. Kim NGRH, Li LS, Kim H, Lee JS, Kim JH, Kim NK, Kim H. Identification of MARCKS, FL11383 and TAF1B as putative novel target genes in colorectal carcinomas with microsatellite instability. Oncogene. 2002;21:5081-7.

37. Son HY, Hwangbo Y, Yoo SK, Im SW, Yang SD, Kwak SJ, Park MS, Kwak SH, Cho SW, Ryu JS, et al. Genome-wide association and expression quantitative trait loci studies identify multiple susceptibility loci for thyroid cancer. Nat Commun. 2017;8:15966.

38. Gudmundsson J, Thorleifsson G, Sigurdsson JK, Stefansdottir L, Jonasson JG, Gudjonsson SA, Gudbjartsson DF, Masson G, Johannsdottir H, Halldorsson GH, et al. A genome-wide association study yields five novel thyroid cancer risk loci. Nat Commun. 2017;8:14517.

39. Snyder JT, Rossman KL, Baumeister MA, Pruitt WM, Siderovski DP, Der CJ, Lemmon MA, Sondek J. Quantitative analysis of the effect of phosphoinositide interactions on the function of $\mathrm{Dbl}$ family proteins. J Biol Chem. 2001;276:45868-75

40. Zhang YJ, Wen CL, Qin YX, Tang XM, Shi MM, Shen BY, Fang Y. Establishment of a human primary pancreatic cancer mouse model to examine and investigate gemcitabine resistance. Oncol Rep. 2017;38:3335-46.

41. Wang Z, Zhang J, Ye M, Zhu M, Zhang B, Roy M, Liu J, An X. Tumor suppressor role of protein 4.1B/DAL-1. Cell Mol Life Sci. 2014;71:4815-30.

42. Sanuki R, Watanabe S, Sugita Y, Irie S, Kozuka T, Shimada M, Ueno S, Usukura J, Furukawa T. Protein-4.1G-mediated membrane trafficking is essential for correct rod synaptic location in the retina and for normal visual function. Cell Rep. 2015;10:796-808.

43. Ji Z, Shi X, Liu X, Shi Y, Zhou Q, Liu X, Li L, Ji X, Gao Y, Qi Y, Kang Q. The membrane-cytoskeletal protein $4.1 \mathrm{~N}$ is involved in the process of cell adhesion, migration and invasion of breast cancer cells. Exp Ther Med. 2012:4:736-40

44. Feng G, Guo K, Yan Q, Ye Y, Shen M, Ruan S, Qiu S. Expression of protein 4.1 family in breast cancer: database mining for 4.1 family members in malignancies. Med Sci Monit. 2019;25:3374-89.

45. Robb VALW, Gascard P, Perry A, Mohandas N, Gutmann DH. Identification of a third Protein 4.1 tumor suppressor, Protein 4.1R, in meningioma pathogenesis. Neurobiol Dis. 2003;13:191-202. 
46. Schulz WAIM, Djuidje CE, Hader C, Rahnenführer J, Engers R. Changes in cortical cytoskeletal and extracellular matrix gene expression in prostate cancer are related to oncogenic ERG deregulation. BMC Cancer. 2010;10:505.

47. Yang X, Yu D, Ren Y, Wei J, Pan W, Zhou C, Zhou L, Liu Y, Yang M. Integrative functional genomics implicates EPB41 dysregulation in hepatocellular carcinoma risk. Am J Hum Genet. 2016;99:275-86.

48. Tontonoz P, Spiegelman BM. Fat and beyond: the diverse biology of PPARgamma. Annu Rev Biochem. 2008:77:289-312

49. Glass CK, Saijo K. Nuclear receptor transrepression pathways that regulate inflammation in macrophages and T cells. Nat Rev Immunol. 2010;10:365-76

50. Korpal M, Puyang X, Jeremy WuZ, Seiler R, Furman C, Oo HZ, Seiler M, Irwin S, Subramanian V, Julie Joshi J, et al. Evasion of immunosurveillance by genomic alterations of PPARgamma/RXRalpha in bladder cancer. Nat Commun. 2017:8:103.

51. Liu F, Sun J, Yu W, Jiang Q, Pan M, Xu Z, Mo F, Liu X. Quantum dot-pulsed dendritic cell vaccines plus macrophage polarization for amplified cancer immunotherapy. Biomaterials. 2020;242:119928.
52. Kargl J, Zhu X, Zhang H, Yang GHY, Friesen TJ, Shipley M, Maeda DY, Zebala JA, McKay-Fleisch J, Meredith G, et al. Neutrophil content predicts lymphocyte depletion and anti-PD1 treatment failure in NSCLC. JCI Insight. 2019;4:e130850.

53. Oldford SA, Marshall JS. Mast cells as targets for immunotherapy of solid tumors. Mol Immunol. 2015;63:113-24.

54. Savage PA, Leventhal DS, Malchow S. Shaping the repertoire of tumorinfiltrating effector and regulatory T cells. Immunol Rev. 2014;259:245-58.

55. Widschwendter M, Fiegl H, Egle D, Mueller-Holzner E, Spizzo G, Marth C, Weisenberger DJ, Campan M, Young J, Jacobs I, Laird PW. Epigenetic stem cell signature in cancer. Nat Genet. 2007;39:157-8.

\section{Publisher's Note}

Springer Nature remains neutral with regard to jurisdictional claims in published maps and institutional affiliations.
Ready to submit your research? Choose BMC and benefit from:

- fast, convenient online submission

- thorough peer review by experienced researchers in your field

- rapid publication on acceptance

- support for research data, including large and complex data types

- gold Open Access which fosters wider collaboration and increased citations

- maximum visibility for your research: over $100 \mathrm{M}$ website views per year

At BMC, research is always in progress.

Learn more biomedcentral.com/submissions 